Progressive dyspnoea

\section{A rare cause of dyspnoea}

\section{Surya Prakash Bhatt, T K Luqman-Arafath, Anant Mohan, Randeep Guleria}

\begin{abstract}
A 51-year-old male non-smoker presented with a complaint of progressive dyspnoea over 25 years, worsening during the past year. There was occasional cough with mucoid expectoration. There was no history of fever, cutaneous rashes, joint pains, Raynaud's phenomenon or chronic drug intake. There was no history of exposure to occupational dusts. The patient had been treated for pulmonary tuberculosis in the past with no improvement in symptoms. On physical examination, he was of thin build and dyspnoeic at rest with a respiratory rate of 30/min. He had grade 3 digital clubbing. He did not have pallor, cyanosis or pedal oedema. On auscultation of the chest, he had vesicular breathing with bilateral basal fine end-inspiratory crepitations. He was on inhaled bronchodilators and home oxygen therapy.

Baseline investigations revealed haemoglobin of $17 \mathrm{~g} / \mathrm{dl}$, haematocrit $50.7 \%$ and a total leucocyte count of $9600 / \mathrm{mm}^{3}$. Erythrocyte sedimentation rate at the end of the first hour (Westergren method) was $35 \mathrm{~mm}$. Chest radiograph and high resolution chest tomography (HRCT) images are shown in figs 1 and 2 . Arterial blood gas analysis showed type
\end{abstract}

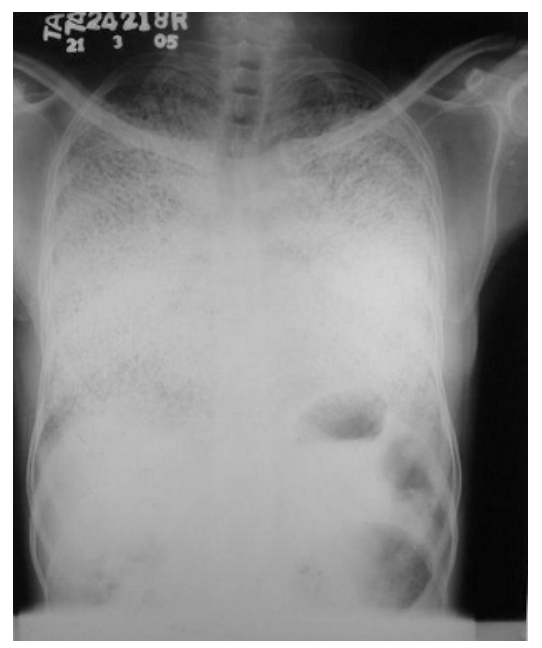

Figure 1 Chest radiograph showing widespread diffuse haziness with nodules in the upper zones, completely obscuring the heart borders and diaphragm.
1 respiratory failure. Spirometry showed a severe restrictive abnormality with impaired diffusion capacity of the lung. An echocardiogram showed left ventricular hypertrophy and no evidence of cor pulmonale.

\section{QUESTIONS}

1. Describe the chest radiograph and HRCT findings

2. What is the diagnosis and how would you confirm it?

3. What is the usual course of the disease and how is it the managed?

\section{ANSWERS}

1. Describe the chest radiograph and HRCT findings

The chest radiograph shows diffuse haziness with fine reticulo-nodular shadows throughout both lung fields, with relative sparing of the apices (fig 1). The heart borders and diaphragm are obscured. HRCT of the chest reveals bilateral interstitial fibrosis with extensive intra-alveolar calcifications with relative apical sparing (fig 2). There is no pleural thickening and there are no subpleural cysts. The pulmonary vessels and heart do not show any abnormalities.

2. What is the diagnosis and how would you confirm it?

The typical dissociation between radiological and clinical features with

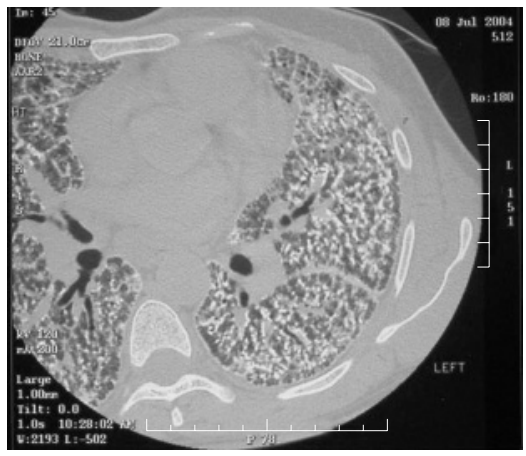

Figure 2 High resolution computed tomograph of the chest showing widespread extensive calcific nodules with thickening of the interlobular pleura. widespread calcific nodules on chest radiograph should make the diagnosis simple. However, other common differential diagnoses with this dissociation, such as sarcoidosis, silicosis and anthracosis, should be considered. Indeed, this rare disease is frequently mistaken for miliary tuberculosis. The predominant basal involvement with relative apical sparing should help differentiate pulmonary alveolar microlithiasis (PAM) from pulmonary Langerhans' cell histiocytosis. Biopsy is the gold standard for diagnosis. Sputum or bronchial lavage analysis showing microliths and CT guided aspiration cytology can also be diagnostic.

3. What is the usual course of the disease and how is it managed?

The clinical course of the disease is slow but progressive, ending in respiratory failure and cor pulmonale. Treatment is palliative and consists of oxygen therapy. Bisphosphonates, oral steroids and therapeutic broncho-alveolar lavage have been tried with unconvincing results. The inability to find a pathological model has made therapy difficult.

\section{DISCUSSION}

PAM is a rare chronic lung disease of unknown aetiology characterised by widespread deposition of laminated intra-alveolar calcospherites, in the absence of any identified disorder of calcium metabolism. To date, 576 cases have been reported. ${ }^{1}$ Although cases have been reported at both age extremes, most patients are in the third or fourth decade when diagnosed. ${ }^{2}$ There is a male predominance with three fifths of cases occurring in males. ${ }^{2}$

The aetio-pathogenesis remains unknown. PAM usually occurs sporadically, although half the patients have a family member affected, usually a sibling. ${ }^{3}$ Recent reports from Turkey of cases in siblings and several families with parental consanguinity have suggested an autosomal recessive mode of inheritance. ${ }^{45}$ It is also possible that environmental factors may play a role in the onset of disease in genetically predisposed individuals. ${ }^{6}$ There are two major hypotheses regarding the aetio-pathogenesis of PAM. One, it is an inherited metabolic abnormality involving the enzyme carbonic anhydrase localised to the alveolar surface, resulting in increased alkalinity of the alveolar surface and resultant precipitation of calcareous salts. ${ }^{7}$ Two, it is due to impaired mucociliary clearance resulting in accumulation of mucus in the bronchoalveolar tree and resultant calcification around these mucus deposits. ${ }^{89}$ On light microscopy, alveoli contain periodic acid Schiff positive calcospherites, which are laminated calcium phosphate concretions 
with small amounts of magnesium and aluminium.

Most cases are diagnosed incidentally due to chest radiograph findings, and when symptomatic the disease commonly manifests with cough and exertional dyspnea. Progression of disease is generally slow, and in the end stages patients can develop respiratory failure and cor pulmonale. Spirometry may reveal normal results or a restrictive pattern with impaired diffusing capacity. The chest radiograph shows bilateral sand-like nodules, which are microliths or calcospherites, usually less than $1 \mathrm{~mm}$ in diameter, creating the typical "sandstorm" pattern. The distribution of these nodular opacities is generally more at the bases disappearing into the apices, or may be more at the hilum and disappearing into the periphery, or rarely along the bronchioles and vessels. There maybe associated sub-pleural bullae, especially at the apices, causing a "black pleural line $\mathrm{e}^{\prime \prime} .{ }^{10}$ Asymptomatic patients may have extensive lesions on chest radiograph which itself is diagnostic in most cases. In cases where further characterisation is required, HRCT shows widespread nodules with calcification and minimal interstitial fibrosis. Calcifications are most prominent at the bases, peripherally, around the mediastinum and near the fissures separating the lobes. As a result, each lobe appears to be surrounded by a fine dense outline - the "stony lung". ${ }^{2}$ HRCT may also reveal ground glass lesions alongside the calcifications, as well as pleural calcification. $^{11}$

\section{FINAL DIAGNOSIS}

The final diagnosis is pulmonary alveolar microlithiasis (PAM).

Postgrad Med J 2007;83:e2 (http://www postgradmedi.com/cgi/content/full/83/976/ e2).

doi: $10.1136 /$ pgmi.2006.050351

\section{............................}

\section{Authors' affiliations}

S P Bhatt, T K Luqman-Arafath, A Mohan,

R Guleria, Department of Internal Medicine, All India Institute of Medical Sciences, Ansarinagar New Delhi, India

Correspondence to: Professor Randeep Guleria, Department of Internal Medicine, All India Institute of Medical Sciences, Ansarinagar, New Delhi 110029, India; randeepguleria2002@ yahoo.com

Received 8 June 2006

Accepted 7 August 2006
Competing interests: None declared.

\section{REFERENCES}

1 Mariotta S, Ricci A, Papale M, et al. Pulmonary alveolar microlithiasis: report on 576 cases published in the literature. Sarcoidosis Vasc Diffuse Lung Dis 2004:21(3):173-81.

2 Castellana G, Lamorgese V. Pulmonary alveolar microlithiasis. Respiration 2003;70:549-55.

3 Sosman MC, Dodd GD, Jones WD, et al. The familial occurrence of pulmonary alveolar microlithiasis. AJR Am J Roentgenol 1957;77:947-1012.

4 Uçan ES, Keyf Al, Aydilek R, et al. Pulmonary alveolar microlithiasis: review of Turkish reports. Thorax 1993;48:171-3.

5 Castellana G, Gentile M, Castellana R, et al. Pulmonary alveolar microlithiasis: clinical features, evolution of the phenotype, and review of the literature. Am J Med Genet 2002;111(2):220-4.

6 Esguerra-Gomez G, Lichtemberger E, Santa-Maria A, et al. Familial pulmonary alveolar microlithiasis: four cases from Columbia. Radiology 1959;72:550-61

7 Lopez-Areal L, Zumarraga R, Gil-Turner C, et al. Microlithiasis alveolar pulmonary familiar $\mathrm{e}$ infantile. Rev Clin Esp 1965;97:389-95.

8 D'Addabbo A, Fratello A, Fanfani G, et al. Lung scanning and clearance of inhaled radiogold (198Au) particles in three patients with microlithiasis. Rofo 1981;135:296-300

9 Chinacoti N, Thsanghai P. Pulmonary alveolar microlithiasis associated with the inhalation of snuff in Thailand. Dis Chest 1957:22:687-9.

10 Felson B. Thoracic calcifications. Dis Chest 1969;56:336-43

11 Sumikawa $\mathrm{H}$, Johkoh T, Tomiyama N, et al. Pulmonary alveolar microlithiasis: $\mathrm{CT}$ and pathologic findings in 10 patients. Monaldi Arch Chest Dis 2005;63(1):59-64 